\title{
Crystal Phases and Phase Transitions in a Highly Polymorphogenic Molecular Gyroscopes with meta-Methoxytrityl Frames
}

Jose Nuñez, Tinh-Alfredo V. KhuongLuis M. Campos, Norberto Farfán, Hung Dang, Steve Karlen and Miguel A. Garcia-Garibay*

University of California, Los Angeles, Department of Chemistry and Biochemistry, 405 Hilgard Ave., Los Angeles, CA 90095-1569

\section{Supplementary Information}

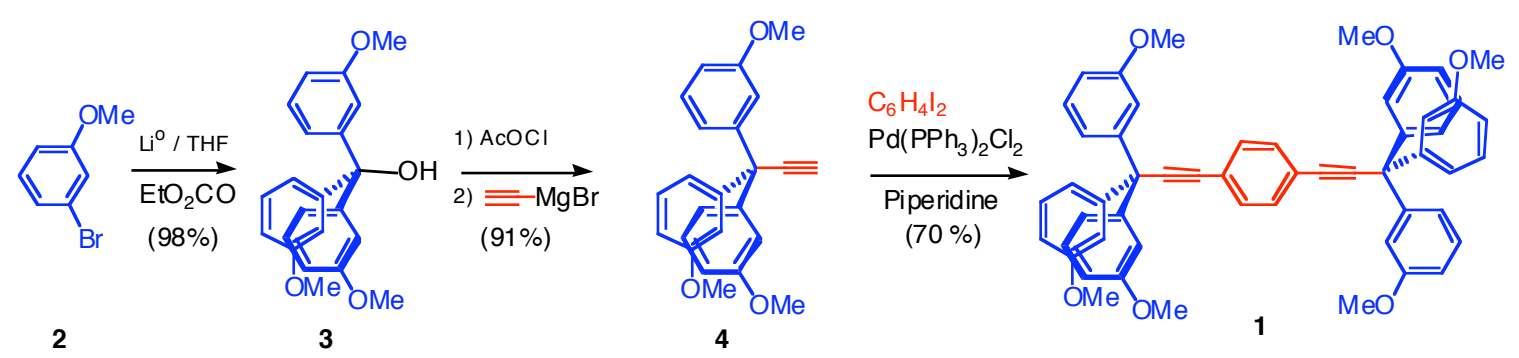

Synthesis of alcohol 3. A solution of $230 \mathrm{~mL}$ of THF and $7.5 \mathrm{~g}(40.1 \mathrm{mmol})$ metabromoanisole 2 were treated with $0.85 \mathrm{~g}(122.5 \mathrm{mmol}) \mathrm{Li}$ metal at room temperature. Upon completion, the anion was reacted with $1.67 \mathrm{~mL}(13.8 \mathrm{mmol})$ diethyl carbonate in THF for 12 $\mathrm{h}$ at room temperature. The reaction mixture was worked-up with sat'd ammonium chloride solution and extracted 3 times with $100 \mathrm{~mL}$ of DCM. Silica gel chromatography using 95:5 $\mathrm{v} / \mathrm{v}$ of hexanes/ether as eluant gave alcohol $\mathbf{3}$ as a colorless crystalline solid in $89 \%$ isolated yield. mp: $118-119^{\circ} \mathrm{C}$ IR (KBr): 2960, 2826, 1597, 1486, 1452, 1445, 1427, 1330, 1297, 1252, 1169, 1040, 862, 770, 703. ${ }^{1} \mathrm{H}$ NMR (500 MHz, $\left.\mathrm{CDCl}_{3}\right)$ : $\square 7.23$ (dd, J=8.0, 3H), 6.97 (dd, J=2.2, 2.2, 3H), $6.88(\mathrm{~d}, \mathrm{~J}=7.8,3 \mathrm{H}), 6.83(\mathrm{~d}, \mathrm{~J}=8.4,2.2,3 \mathrm{H}), 3.73(\mathrm{~s}, 9 \mathrm{H}), 3.26$ (bs, 1H, OH). ${ }^{13} \mathrm{C}$ NMR (125 MHz, $\left.\mathrm{CDCl}_{3}\right): \square 159.0,148.2,128.6,120.4,113.8,112.3,81.6,54.9$.

\footnotetext{
${ }^{\ddagger}$ Permanent address: Departamento de Quimica, Centro de Investigacion y de Estudios Avanzados del IPN, Mexico D.F., 07000
} 
Synthesis of alkyne 4. A solution of $2.6 \mathrm{~g}(7.4 \mathrm{mmol})$ of alcohol $\mathbf{3}$ in excess acetyl chloride was stirred for $3 \mathrm{~h}$ at room temperature under argon. Removal of the excess acetyl choride by evaporation was followed by washing with $20 \mathrm{~mL}$ of benzene giving the chloride which was dissolved in benzene and subsequently reacted with $68 \mathrm{~mL}(34 \mathrm{mmol})$ ethynylmagnesium bromide in THF also at room temp for $12 \mathrm{~h}$. Silica gel chromatography using a 9:1 mixture $\mathrm{v} / \mathrm{v}$ of hexanes/ether as eluant gave the alkyne in $91 \%$ yield. HRMS: $\mathrm{C}_{24} \mathrm{H}_{22} \mathrm{O}_{3}$, Exact Mass: 358.1569 Found (MALDI): 358.1554. IR (KBr): 3283, 3074, 3001, 2956, 2938, 2934, 2093, 1933, 1847, 1715, 1599, 1584, 1486, 1464, 1433, 1316, 1291, 1251, 1186, 1150, 1093, 1051, 997, 966, 878, 857, 770, 698, 642. ${ }^{1} \mathrm{H}$ NMR (500 MHz, $\left.\mathrm{CDCl}_{3}\right):$ $7.22(\mathrm{dd}, \mathrm{J}=8.0,3 \mathrm{H}), 6.89$ (dd, J=2.1, 2.1, 3H), $6.81(\mathrm{~d}, \mathrm{~J}=8.0,3 \mathrm{H}), 6.77$ (dd, J=8.0, 2.1, 3H), 3.73 (s, 9H), $2.74(\mathrm{~s}, 1 \mathrm{H}) .{ }^{13} \mathrm{C} \mathrm{NMR}\left(125 \mathrm{MHz}, \mathrm{CDCl}_{3}\right)$ : $\square 159.2,146.0,128.8,121.6,115.4$, $112.0,89.4,73.5,55.5,55.1 \mathrm{~cm}^{-1}$.

Synthesis of gyroscope 1. To a solution of $1.83 \mathrm{~g}(5.1 \mathrm{mmol})$ alkyne $4 \mathrm{in} 25 \mathrm{~mL}$ of piperidine, $0.85 \mathrm{~g}(0.95 \mathrm{mmol})$ of 1,4-diiodobenzene, $0.26 \mathrm{~g}(0.37 \mathrm{mmol})$ of $\mathrm{Pd}\left(\mathrm{PPh}_{3}\right)_{2} \mathrm{Cl}_{2}$ and $0.18 \mathrm{~g}(0.95 \mathrm{mmol})$ of $\mathrm{CuI}$ was added. The mixture was heated to $80{ }^{\circ} \mathrm{C}$ for $6 \mathrm{~h}$ and worked up with sat'd ammonium chloride solution. The crude material was extracted with DCM and dried over anhydrous magnesium sulfate. Purification by silica gel chromatography using 7:3 $\mathrm{v} / \mathrm{v}$ hexanes/ether gave the white microcrystalline product in $70 \%$ yield. $\mathrm{mp}: 180-181^{\circ} \mathrm{C}$; HRMS: $\mathrm{C}_{54} \mathrm{H}_{46} \mathrm{O}_{6}$ Exact Mass: 790.3294 Found (MALDI): 790.3297; IR: 3003, 2955, 2938, 2834, 1930, 1844, 1755, 1599, 1583, 1487, 1464, 1450, 1433, 1314, 1291, 1250, 1185, 1150, 1094, 1051, 997, 965, 887, 858, 839, 769, 724, 698, 667. ${ }^{1} \mathrm{H}$ NMR (500 MHz, $\left.\mathrm{CDCl}_{3}\right)$ : $\square 7.43$ (s, 4H), $7.22(\mathrm{dd}, \mathrm{J}=8.0,6 \mathrm{H}), 6.93(\mathrm{dd}, \mathrm{J}=1.8,2.0,6 \mathrm{H}), 6.88$ (d, J=8.0, 6H), 6.80 (dd, J=2.0, 
8.0, 6H), $3.74(\mathrm{~s}, 18 \mathrm{H}) .{ }^{13} \mathrm{C}$ NMR $\left(500 \mathrm{MHz}, \mathrm{CDCl}_{3}\right): \square 159.3,146.5,131.4,128.9,123.2$, 121.7, 115.4, 112.0, 97.1, 84.9, 56.2, 55.1. 


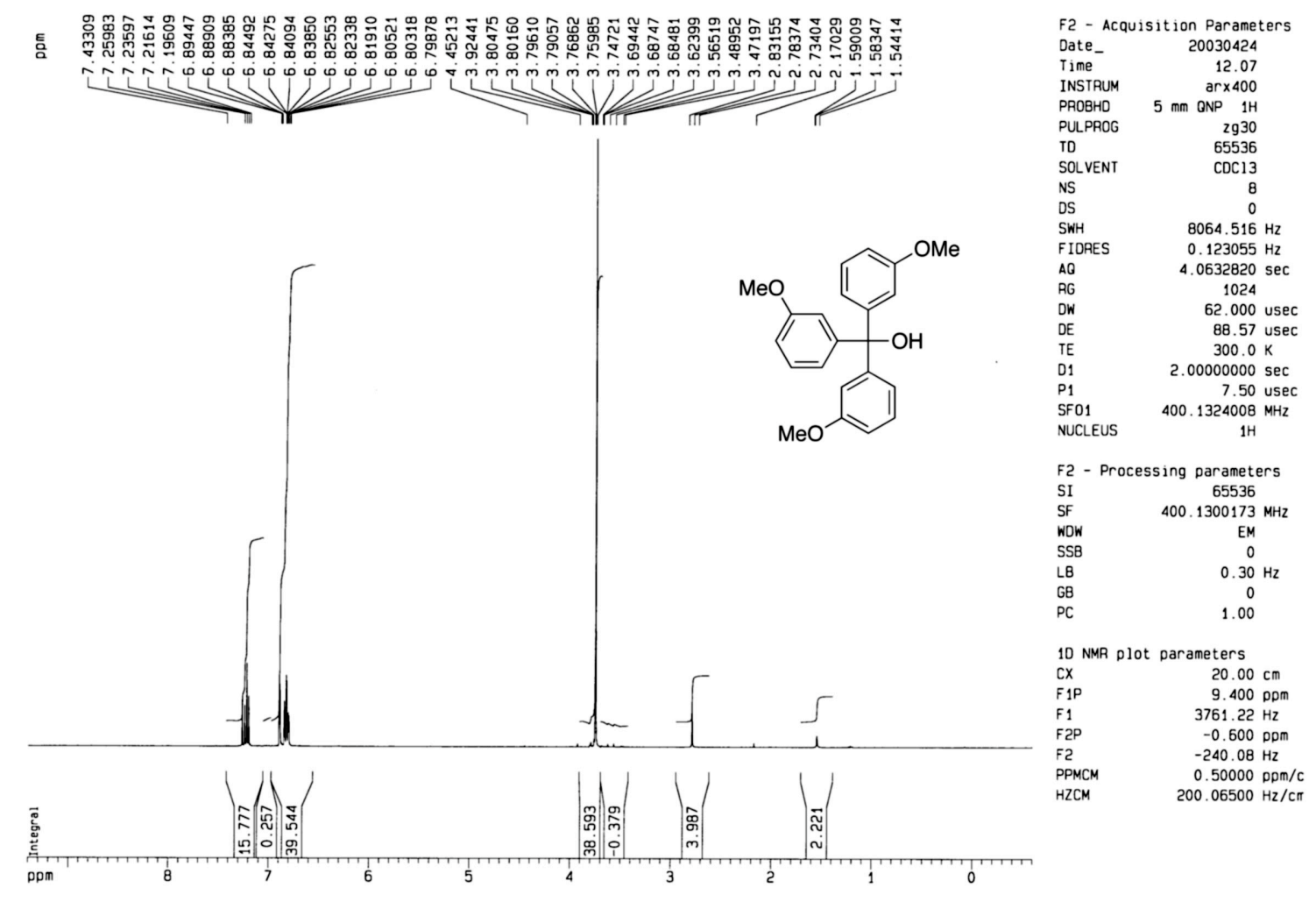



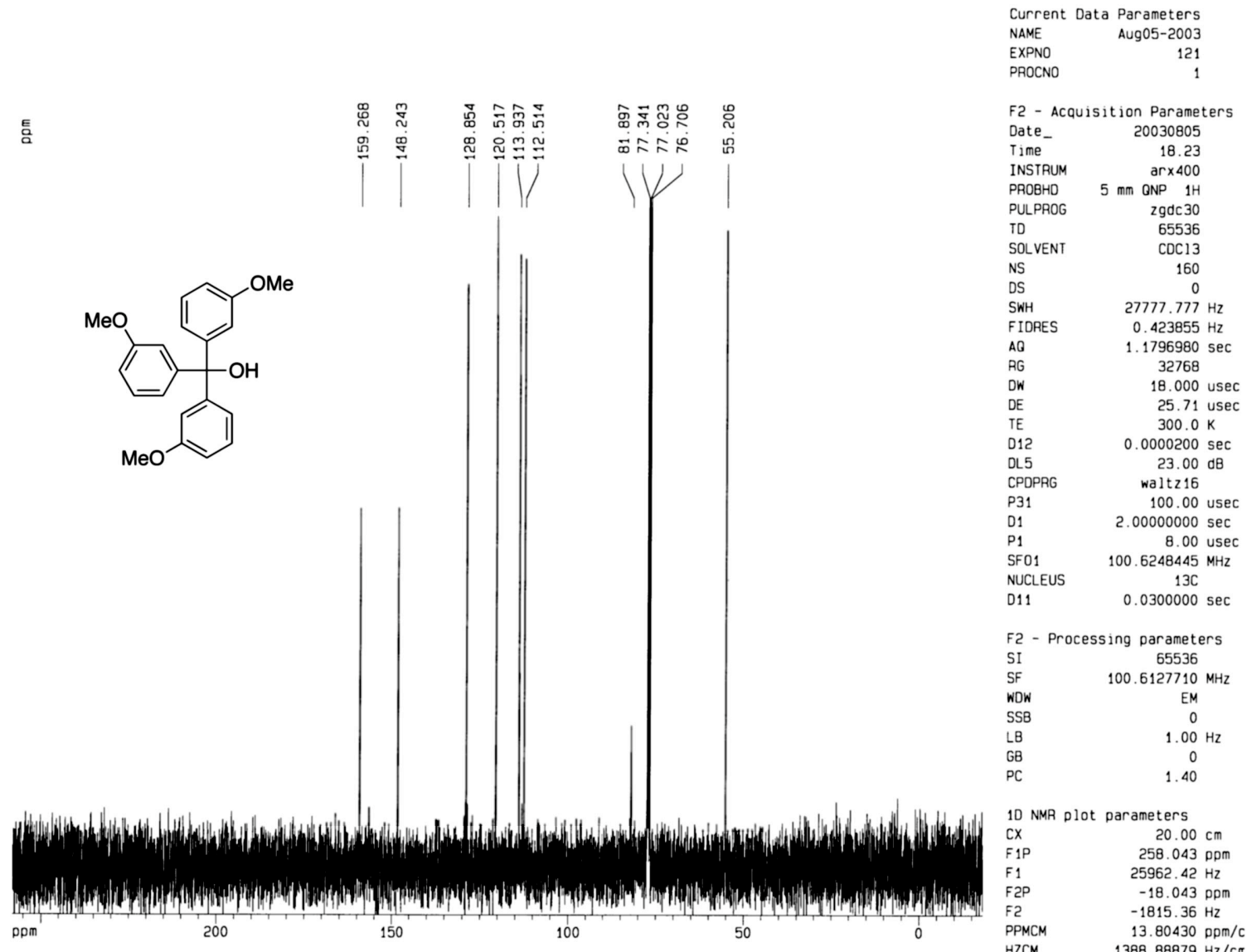

10 NMR plot parameters

$\begin{array}{lr}\text { CX } & 20.00 \mathrm{~cm} \\ \text { F1P } & 258.043 \mathrm{ppm} \\ \text { F1 } & 25962.42 \mathrm{~Hz} \\ \text { F2P } & -18.043 \mathrm{ppm} \\ \text { F2 } & -1815.36 \mathrm{~Hz} \\ \text { PPMCM } & 13.80430 \mathrm{ppm} / \mathrm{c} \\ \text { HZCM } & 1388.88879 \mathrm{~Hz} / \mathrm{cm}\end{array}$




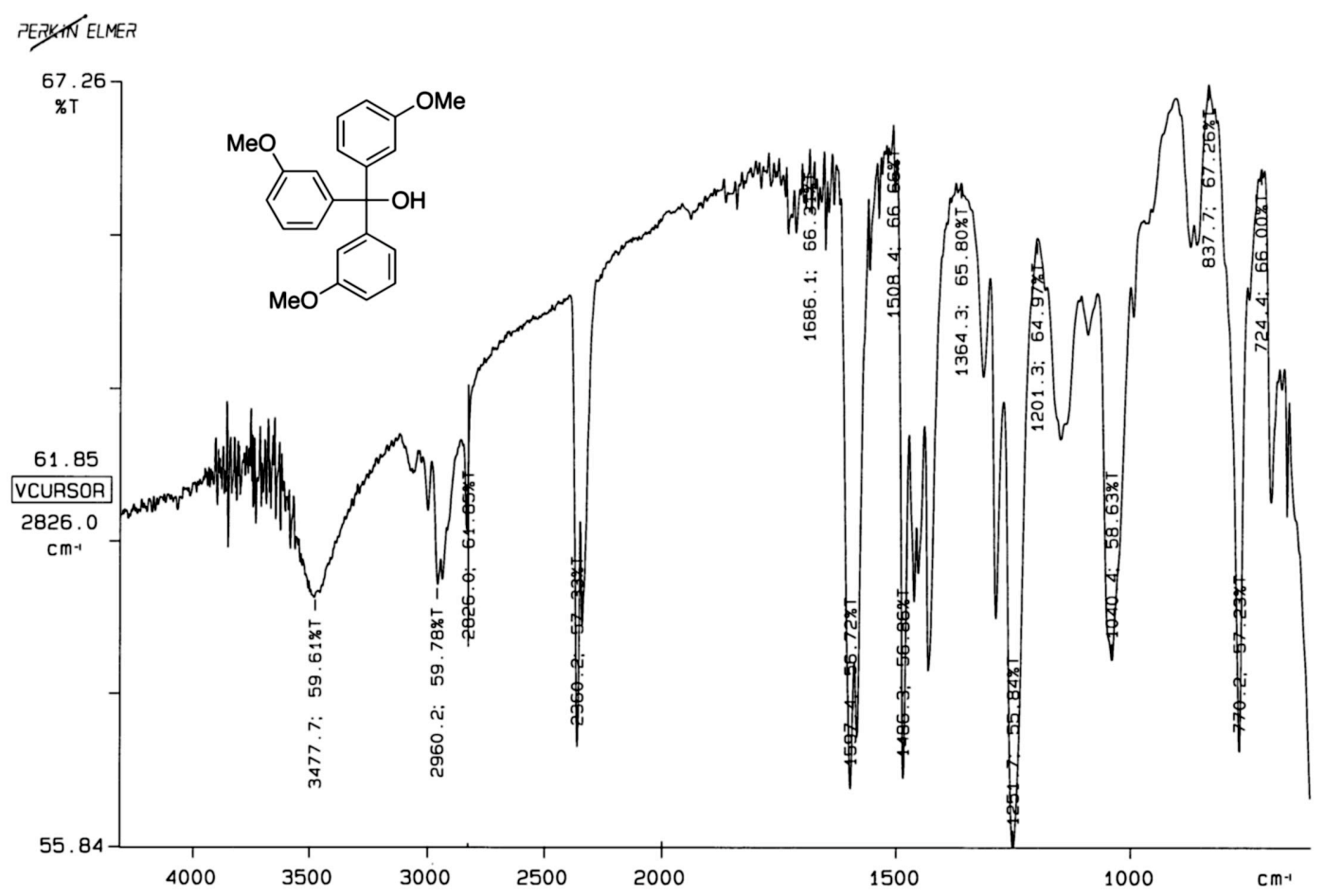




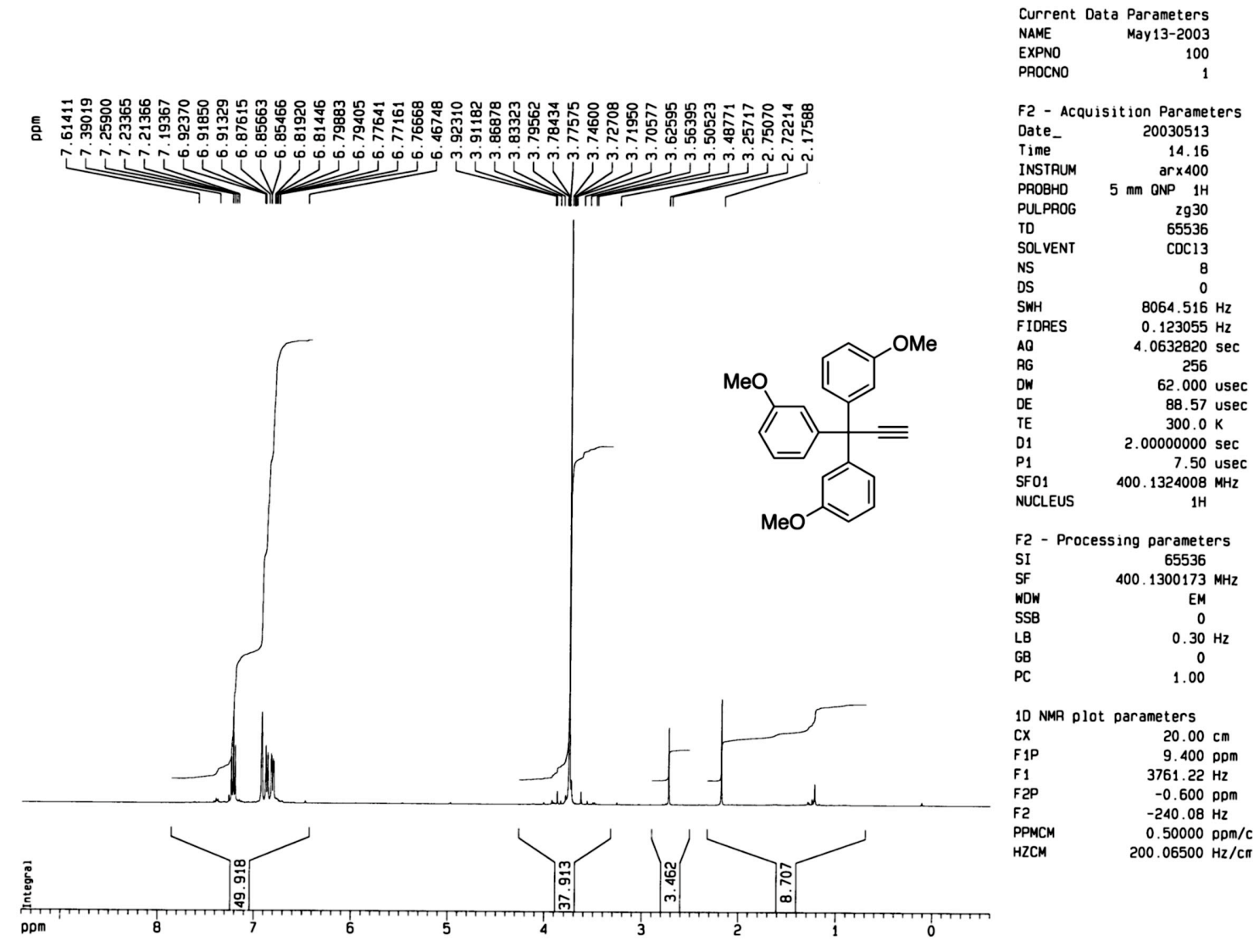




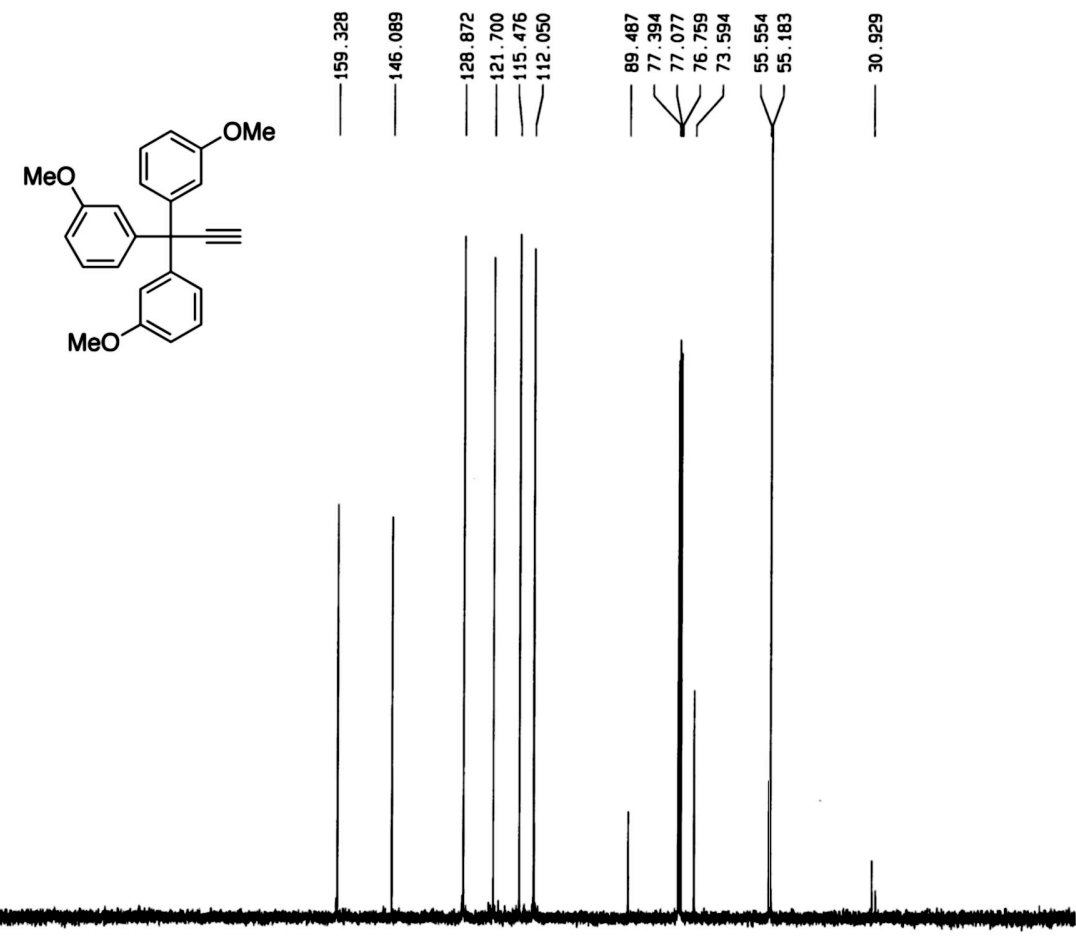

$\begin{array}{ll}\text { Current Data Parameters } \\ \text { NAME } & \text { May13-2003 }\end{array}$

May 13-2003

PROCNO

F2 - Acquisition Parameter

Date_ 20030513

Time 14.25

INSTRUM $\quad$ ar $\times 400$

PROBHD $5 \mathrm{~mm}$ ONP $1 \mathrm{H}$

$29 d c 30$

SOLVENT $\quad \mathrm{COC} 13$

$\begin{array}{lr}\text { NS } & 128 \\ \text { DS } & 0\end{array}$

$\begin{array}{ll}\text { OS } & 0 \\ \text { SWH } & 27777.777 \mathrm{~Hz}\end{array}$

IDRES $\quad 0.423855 \mathrm{~Hz}$

$\begin{array}{cc}\text { AQ } & 1.1796980 \mathrm{sec} \\ \text { RG } & 32768\end{array}$

DW 18.000 usec

$\begin{array}{ll}\text { DE } & 25.71 \text { usec } \\ \text { TE } & 300.0 \mathrm{~K}\end{array}$

$012 \quad 0.0000200 \mathrm{sec}$

OL5 $23.00 \mathrm{~dB}$

CPDPAG waltz16

P31 100.00 usec

D1 $2.00000000 \mathrm{sec}$

SF01 $\quad 100.6248445 \mathrm{MHz}$

NUCLEUS $\quad-100.6248445 \mathrm{MH}$

$\begin{array}{lc}\text { NUCLEUS } & 13 \mathrm{C} \\ \text { D11 } & 0.0300000 \mathrm{sec}\end{array}$

F2 - Processing parameters

SI $\quad 65536$

SF $\quad 100.6127710 \mathrm{MHz}$

WOW EM

$\begin{array}{lc}\text { SSB } & 0 \\ \text { LB } & 1.00 \mathrm{~Hz}\end{array}$

$\begin{array}{lr}\text { GB } & 0 \\ P C & 1.40\end{array}$

10 NMR plot parameters

$\begin{array}{lr}C X & 20.00 \mathrm{~cm} \\ F & \end{array}$

$\begin{array}{ll}\mathrm{F} 1 \mathrm{P} & 258.043 \mathrm{ppm} \\ \mathrm{F} 1 & -18.043 \mathrm{ppm}\end{array}$

$\begin{array}{ll}\text { F2P } & -18.043 \mathrm{ppm}\end{array}$

F2 $-1815.36 \mathrm{~Hz}$

PPMCM $13.80430 \mathrm{ppm} / \mathrm{c}$ $1388.88879 \mathrm{~Hz} / \mathrm{cr}$ 


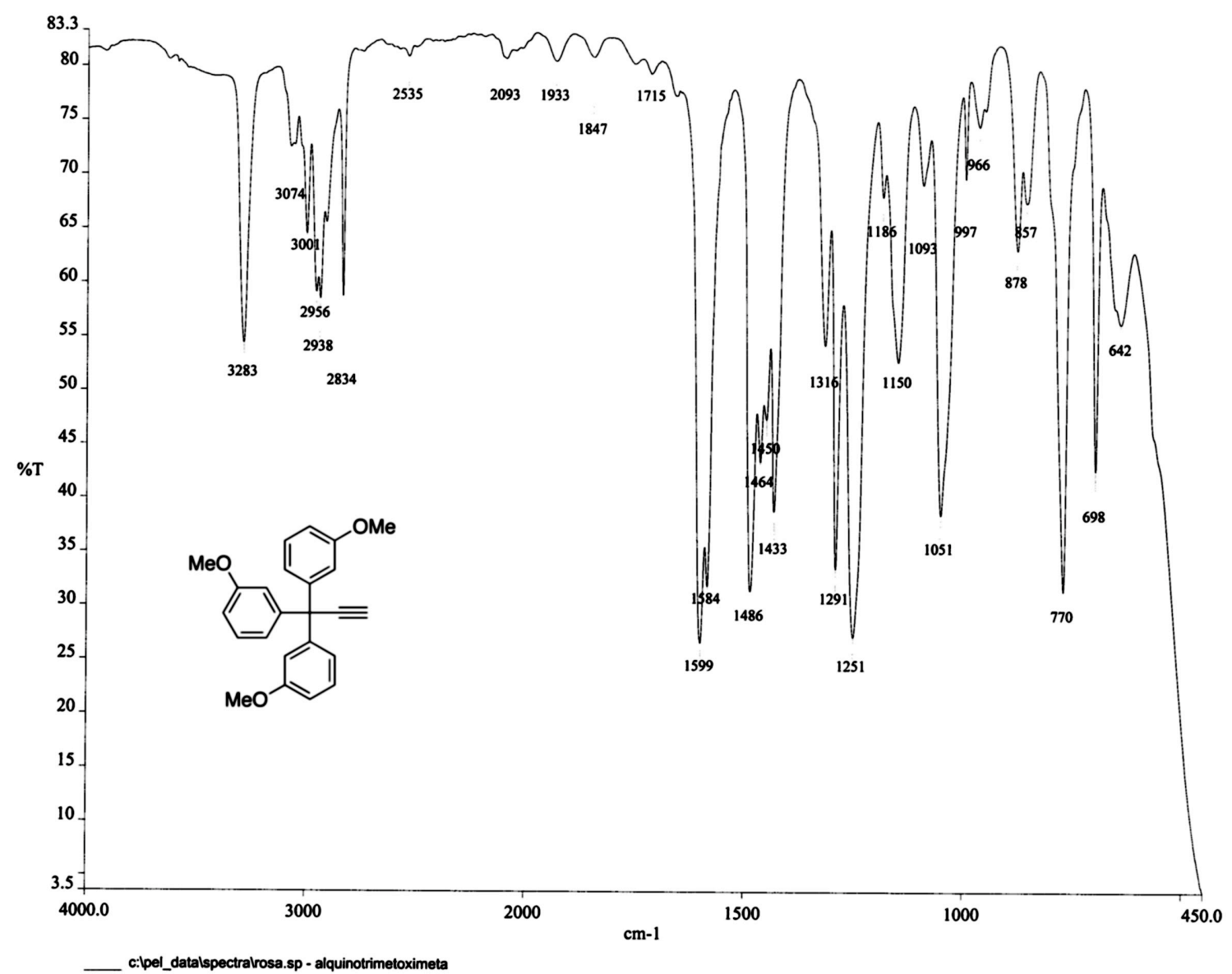




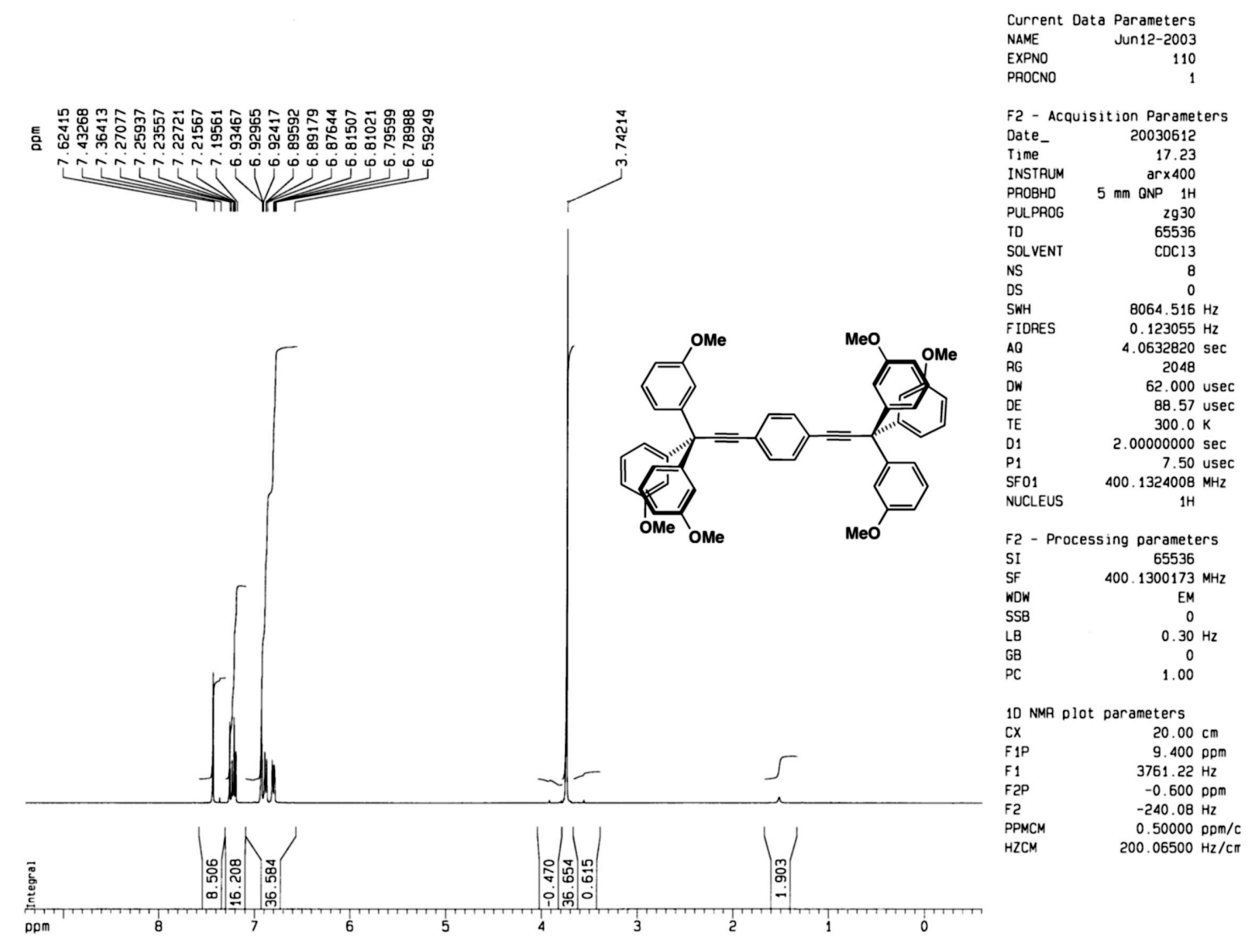




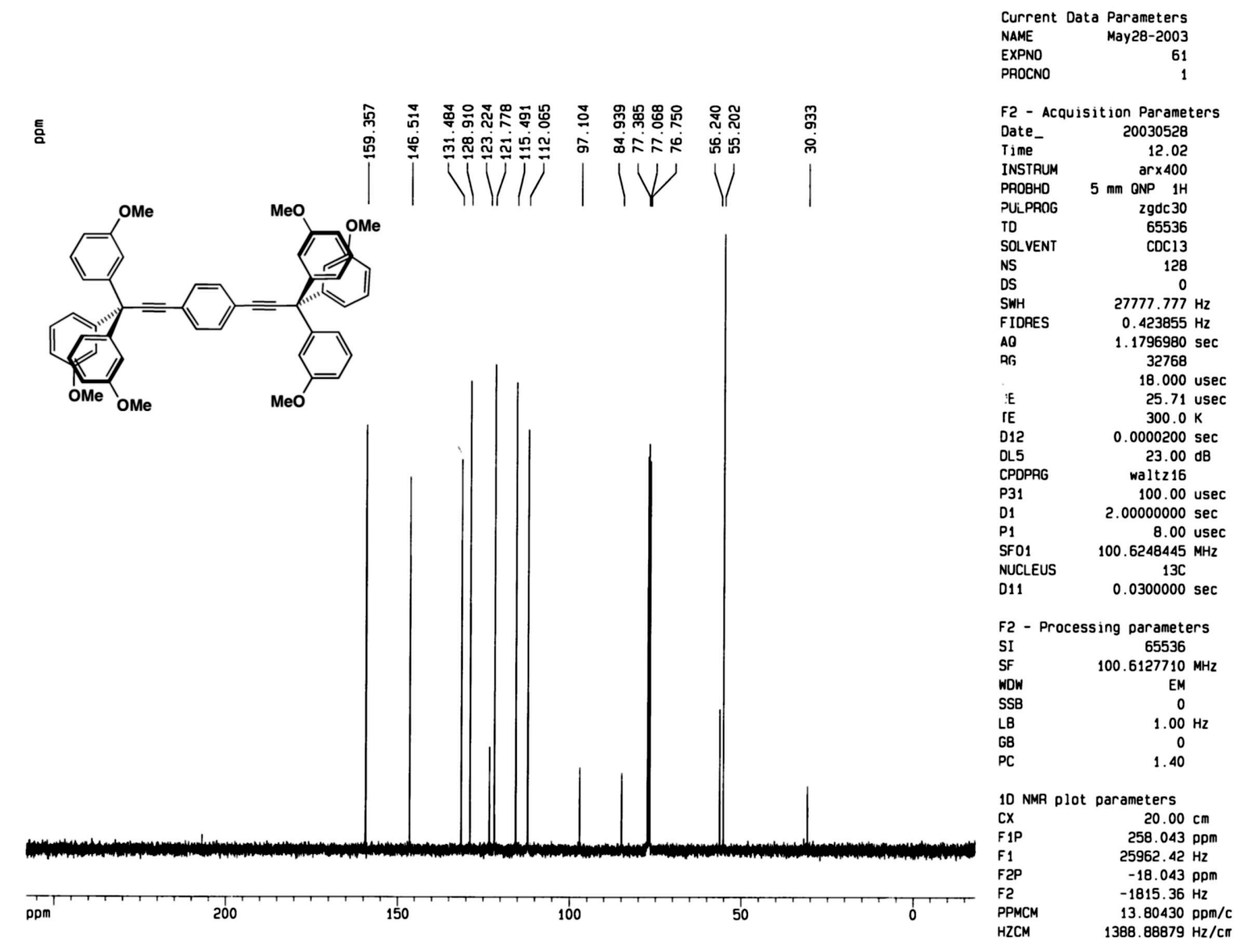




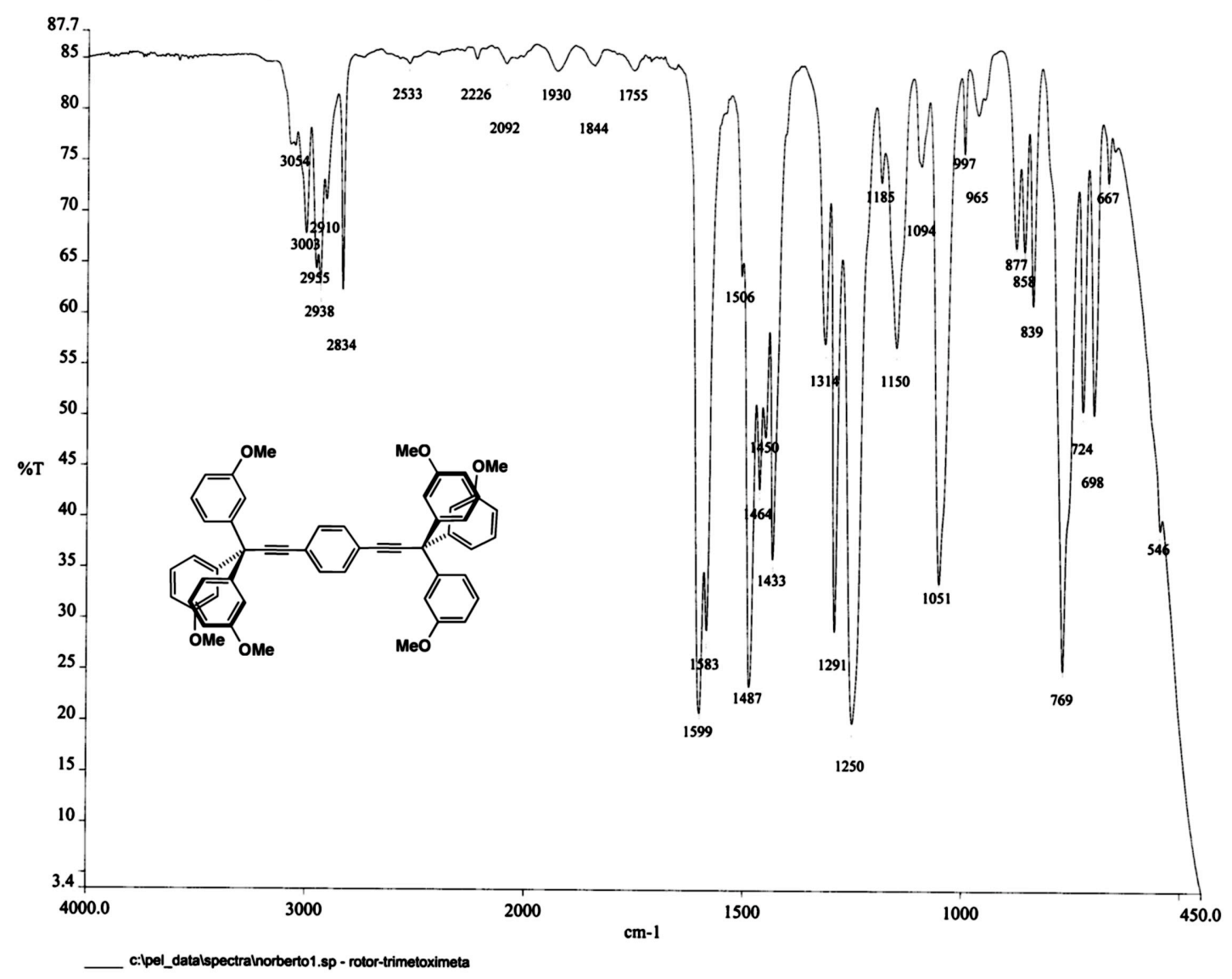

